\title{
Comparison of hemodynamic and clinical outcomes of transcatheter and sutureless aortic bioprostheses: how to make the right choice in intermediate risk patients
}

\author{
Augusto D’Onofrio, Assunta Fabozzo, Gino Gerosa \\ Division of Cardiac Surgery, University of Padova, Padova, Italy \\ Correspondence to: Augusto D’Onofrio, MD, PhD. Division of Cardiac Surgery, University of Padova, Via Giustiniani 2, 35128, Padova, Italy. \\ Email: adonofrio@hotmail.it.
}

\begin{abstract}
Current surgical treatment options for aortic valve stenosis (AS), as alternatives to that of conventional operation with a midline sternotomy, include sutureless valve replacement (SUAVR) and transcatheter valve implantation (TAVI). Patients with high surgical risk, or those who are judged to be inoperable, are typically good candidates for the TAVI procedure. The best treatment option in patients with an intermediate risk profile, however, the so called "grey zone", is still currently under debate. Sutureless aortic valve replacement has been recently presented as a valid alternative for patients with low- to intermediate-risk. Data available on prostheses' hemodynamic performance and patients' clinical outcomes play a crucial role in the process of device selection. Compared to TAVI, SUAVR provides lower rate of significant postoperative paravalvular leak (PVL), which has shown to be a predictor for mortality. On the contrary, transcatheter valves seem to perform better in terms of transvalvular mean and peak gradients. Therefore, SUAVR and TAVI are both reliable options in patients with severe aortic valve stenosis, as an alternative to conventional surgery, and the choice of the best device should be tailored to patient's anatomical and surgical characteristics.
\end{abstract}

Keywords: Transcatheter aortic valve implantation; sutureless aortic valve replacement; valve prosthesis; aortic valve

Submitted Aug 01, 2017. Accepted for publication Sep 05, 2017.

doi: 10.21037/acs.2017.09.04

View this article at: http://dx.doi.org/10.21037/acs.2017.09.04

\section{Introduction}

Technological advances have recently supported procedural changes and improvements in aortic valve replacement by favoring the development of novel surgical approaches and new valve prostheses. Amongst them, sutureless (Perceval; Livanova, London, UK) and rapid-deployment valves (Intuity Valve System; Edwards Lifesciences, Irvine, CA, USA) are successfully used in several settings of conventional and minimally invasive procedures (SUAVR, sutureless aortic valve replacement). They are currently one of the more appealing substitutes for surgeons, who can take advantage of their simplified implantation technique whilst maintaining auxiliary cardiopulmonary bypass and cardioplegic arrest $(1,2)$. On the other hand, transcatheter aortic valves (TAVI) are delivered in a microinvasive fashion $(\mu-\mathrm{ICS}$, in a beating and non-assisted heart) (3) and are considered a treatment option primarily for high-risk patients $(4,5)$. Current guidelines $(6,7)$ and data available from ongoing randomized trials (8) confirm TAVI's potential benefits in some elective patients with intermediate risk. In this paper, we aim to share our perspective on therapeutic approaches for patients with severe aortic stenosis by reviewing hemodynamic data and clinical evidence for SUAVR versus TAVI.

\section{State of art}

In 2016, our group published the results of a multicenter study on clinical and hemodynamic comparisons between SUAVR and TAVI (9). All patients from 33 Italian centers undergoing TAVI (Sapien and Sapien XT), from 2007 to 2012, and all patients who underwent isolated SUAVR 
(Perceval) at six European centers, from 2010 and 2014, were included in the analysis. A propensity-matching process was performed to properly define the population characteristics and the level of patients' risk profiles. Our analysis showed that patients undergoing SUAVR or TAVI procedures have similar clinical outcomes in terms of 30 -day $(2.3 \%$ vs. $3.7 \%, \mathrm{P}=0.39)$ and 1 -year mortality (5.8\% vs. $9.4 \%, \mathrm{P}=0.16)$, stroke $(1.9 \%$ vs. $1.9 \%, \mathrm{P}=0.99)$, bleeding $(18.7 \%$ vs. $16.1 \%, \mathrm{P}=0.48)$, and myocardial infarction $(0.5 \%$ vs. $0.9 \%, \mathrm{P}=0.99)$. Moreover, from the hemodynamic standpoint, TAVI showed significantly lower transaortic mean and peak gradients $(13.7 \pm 6.6$ vs. $11.0 \pm 4.6 \mathrm{mmHg}, \mathrm{P}<0.001$ and $26.7 \pm 12.1$ vs. $20.3 \pm$ $8.1 \mathrm{mmHg}, \mathrm{P}<0.001$, respectively) but a higher incidence of post-procedural paravalvular leak (PVL) (severe: $0.5 \%$ vs. $5.1 \%, \mathrm{P}=0.05$; > mild $0.5 \%$ vs. $5.3 \%, \mathrm{P}<0.001)$. Additionally, SUAVR showed higher device success $(98.6 \%$ vs. $88.8 \%$, $\mathrm{P}<0.001$ ), whereas TAVI was associated with shorter hospital and intensive care unit stay compared to SUAVR (11 vs. 6 days, $\mathrm{P}<0.001$ and 2 vs. 1 days, $\mathrm{P}<0.001)$. The established literature provides few other studies comparing SUAVR and TAVI, that focus on hemodynamic valve performance (mainly transvalvular gradients and PVL) and patients' clinical outcomes. A recently published multicenter study (10) matched patients undergoing conventional aortic valve replacement (AVR), SUAVR with Perceval valve, and TAVI. Although patients in the TAVI group presented lower peak gradients compared to conventional surgery and SUAVR $(14.34 \pm 7.5$ vs. $22.75 \pm 11.7$ vs. $19.52 \pm 12.45$ $\mathrm{mmHg}$, respectively, $\mathrm{P}=0.015$ ), they performed worse in terms of intraoperative aortic regurgitation $(0.5 \% v s$. $1.9 \%$ vs. $8.8 \%, \mathrm{P}=0.028$ ), postoperative pacemaker (PM) implantation $(3.9 \%$ vs. $9.8 \%$ vs. $14.7 \%, \mathrm{P}<0.001)$, and peripheral complications $(0 \%$ vs. $0 \%$ vs. $9.8 \%, \mathrm{P}<0.001)$. Furthermore, the authors showed higher survival rates at 30 days, 1 - and 2-year in non-TAVI patients. A higher incidence of PVL in TAVI $(\mathrm{P}<0.001)$ compared to SUAVR (Perceval Valve) was also found in the Miceli et al. (11) analysis, even though authors did not find statistical significance for postoperative transvalvular gradients or for the incidence of AV-block-requiring PM. No statistical significance was shown in the short term for mortality and neurological events. Additionally, data obtained from other SUAVR devices, such as $3 \mathrm{~F}$ Enable Valve (Medtronic, Minneapolis, MN, USA, no longer available on the market) (12) compared to TAVI, showed that the latter had a larger effective orifice area index $(1.00 \pm 0.30$ vs. $\left.0.76 \pm 0.22 \mathrm{~cm}^{2} / \mathrm{m}^{2} ; \mathrm{P}<0.001\right)$, lower pressure gradients
$(8.14 \pm 4.21$ vs. $10.72 \pm 4.01 \mathrm{mmHg} ; \mathrm{P}=0.006)$, less frequent patient-prosthesis mismatch (PPM) $(30.0 \%$ vs. $67.5 \%$; $\mathrm{P}=0.001)$ but more frequent aortic regurgitation $(87.5 \%$ vs. $20.0 \% ; \mathrm{P}<0.001)$. However, survival in the two groups was comparable after 1.5 -year follow-up (log-rank test, $\mathrm{P}=0.95$ ). Finally, a recent review of literature and meta-analysis comparing TAVI and SUAVR (13) confirmed that SUAVR prostheses have a lower incidence of paravalvular leak, with no difference in perioperative mortality, but better survival rates at $1-(\mathrm{OR}=2.40 ; 95 \% \mathrm{CI}: 1.40-4.11 ; \mathrm{P}<0.01)$ and 2 -year $(\mathrm{OR}=4.62 ; 95 \%$ CI: 2.62-8.12; $\mathrm{P}<0.01)$.

One study directly compared hemodynamic performance of Sapien versus Perceval valves and focused specifically on patients presenting with a small aortic annulus $(\leq 21 \mathrm{~mm})$. The authors confirmed higher gradients in the SUAVR group, but similar postoperative indexed effective orifice area in both SUAVR and TAVI groups (14). It has been shown that the incidence of severe PPM after Perceval implantation approximately ranges from 0 to $11 \%$ (15), after Intuity from $6 \%$ to $15 \%(16,17)$, and $8 \%$ after TAVI (up to $27 \%$ if a moderate degree of PPM is considered) (18). There are no data about the impact of PPM after SUAVR on survival, but it has been shown that PPM after TAVI does not have an impact on late-term survival (18). The major difficulty in interpreting this data arises from the variability of TAVI devices included in these papers, on their retrospective and propensity-matched nature, and on the fact that the only SUAVR prostheses considered are the Perceval Valves (and the withdrawn $3 \mathrm{~F})$. Furthermore, no data are available on the comparison between TAVI and the other commercially-available sutureless prosthesis, the Intuity Valve.

In summary, the available data demonstrate that SUAVR prostheses perform better than TAVI in terms of paravalvular leak. However SUAVR valves seem to have higher postoperative transvalvular gradients, although the real clinical impact of this finding is still to be determined.

\section{Discussion}

Patients with high surgical risk, advanced age, or those judged inoperable are typically good candidates for the TAVI procedure, whereas the appropriateness of this approach in younger patients with a lower risk profile is still under debate (18). In fact, recently published European guidelines (7) recommend TAVI in intermediate risk patients (class Ib, LOE B), but an age $<75$ years still favors conventional surgery and suggests that the choice of the 
intervention must take into account the characteristics of the patients as well as advantages and disadvantages of every valve substitute. Therefore, the crucial aspects in considering the choice of best therapeutic approach and most suitable aortic substitute, especially in patients with intermediate- to low-risk, are: (I) evaluation of the hemodynamic performance (PVL and transvalvular gradients); (II) valve durability; (III) rate of pacemaker implantation; (IV) patients' quality of life and (V) costeffectiveness analysis.

\section{Hemodynamic performance}

Paravalvular leak after TAVI is mostly due to inappropriate annular sizing, suboptimal positioning, and irregular calcium distribution over the valve annulus and leaflets which determines an inhomogeneous valve expansion. PVL has shown to be correlated to increased mortality at 1 year $(19,20)$, especially in patients with preoperative valve stenosis and no regurgitation (with no previous ventricular dilation) (21). For this reason, when it comes to treating intermediate and low risk patients, the occurrence of "more than mild" PVL should unquestionably be avoided. Transcatheter devices specifically designed to solve PVL after TAVI are not currently available and results after closure with other kind of vascular occluders are suboptimal (22). New generation TAVI devices have already been engineered with specific novel features capable of reducing, but still not eliminating, PVL. Conversely, SUAVR requires annular decalcification as in conventional surgery, thus explaining the reduced incidence of PVL observed in comparative studies. PVL after SUAVR is related to wrong valve sizing and is more frequent during the learning phase. As shown, current evidence supports that SUAVR provides significant lower incidence of PVL and therefore it should be, so far, considered superior to TAVI on this matter. However, whilst currently available data demonstrate that SUAVR provides statistically significant higher gradients than TAVI, there is no evidence of a significant clinical impact. In fact, the incidence of PPM is similar after TAVI and SUAVR, in studies focusing only on patients with a small aortic annulus. Furthermore, PPM in TAVI seems not significantly associated to late mortality (23). Possible explanations for lower transaortic gradients in TAVR, compared to SUAVR, are: (I) the surgical technique that clearly differs between TAVI and SU-AVR and its postoperative implications; in particular, anemia, hemodilution, and inflammation may have a role in the increased gradients found at discharge in the SU-AVR group, and (II) TAVR are designed with a circumferential anchoring stent that protrudes into the LVOT, which is also expanded during valve implantation and may explain the lower gradients. On the contrary, Perceval prostheses do not reach the subannular region. While TAVI is considered superior to conventional valve prostheses in terms of PPM (23), there are no data supporting the superiority compared to SUAVR.

\section{Valve durability}

The intermediate risk patient profile often entails a younger patient age and longer life expectancy, which therefore makes prosthesis durability a major concern. Durability in TAVI is related to the biological nature of the valve leaflets and to pre-procedural steps, such as valve crimping and intravalvular balloon inflation, which is essential for a transcatheter delivery. Several reports have been recently published showing specific lesions (transverse fractures and longitudinal cleavages) on pericardial leaflets, especially in balloon-expandable valves (24-27), phenomena that can potentially lead to valve deterioration. Unfortunately, longterm durability data after TAVI are still not available in the literature and just a few reports present results in patients with 5-year follow-up (28) that, for valve prosthesis, are not considered sufficient. For instance, the Toronto stentless porcine valve was withdrawn from the market because it showed initial valve deterioration signs only 7 years after implantation (29). Therefore, for both TAVI and SUAVR, longer follow-up is needed to reach timepoints when valverelated adverse events are more likely to occur.

\section{Rate of pacemaker implantation}

No significant differences are found in the literature in terms of incidence of AV block and pacemaker implantation between SUAVR and TAVI $(8,30,31)$. Although currently available SUAVR devices show similar rates of PM implantation, this is not the case in TAVI devices. There is wide variability in terms of postoperative need for PM implantation among the different TAVI devices, as some prostheses show an incidence of PM implantation as high as $28 \%$ with a rate of new or worsened left bundle branch block of $78 \%$ (32). Pacemaker implantation is associated with several complications (e.g., endocarditis, device replacement, ventricular dyssynchrony) and it has also been related to higher 1-year mortality (33) after TAVI. For 


\begin{tabular}{|c|c|c|c|}
\hline Surgical approach & CS-MICS & CS-MICS & $\mu \mathrm{ICS}$ \\
\hline Invasiveness & $+/-$ & + & ++ \\
\hline Transvalvular gradients & $-/+$ & + & ++ \\
\hline Paravalvular leak & ++ & $+1-$ & - \\
\hline Costs & ++ & $+/-$ & - \\
\hline
\end{tabular}

all these reasons, potential conduction disturbances must be taken into high consideration during the prosthesis selection, especially in intermediate-risk patients.

\section{Quality of life and cost effectiveness analysis}

Finally, quality of life and cost effectiveness analysis must be considered during screening and operative decision processes in patients with AS and intermediate surgical risk. In actuality, patients seem to similarly perceive their quality of life after conventional surgery, SUAVR (especially if performed in minimally invasive fashion) (34), and TAVI at 1- and 2-year follow-up. Nevertheless, procedural costs become comparable to conventional surgery (standard of care) only if hospital length of stay after TAVI lasts 5-6 days less than SAVR $(35,36)$. Table 1 summarizes advantages and disadvantages of all surgical options for patients suffering from severe aortic valve stenosis.

\section{Comments and perspective}

The best strategy to minimize procedural complications, optimize valve hemodynamics and improve patients' clinical outcomes relies on the establishment of a meticulous preoperative planning process. In particular, preoperative high resolution CT scanning enables detailed aortic root and valve analysis (included calcium quantification and mapping) with 3D model reconstruction of the aortic root. Sutureless and transcatheter valves should not be considered as competitors, as they have their own field of application and both are valid and surgical options. In fact, in specialized centers, where case-specific preoperative planning is done and availability of all prostheses is ensured to qualified surgeons, the choice of the most appropriate device is totally unbiased. For instance, in patients with asymmetrical distribution of calcium and/or massive and bulky annular calcification-both morphological predictors of PVL and BAV after TAVI—SUAVR may be a better option. Finally, SUAVR significantly facilitates a minimally invasive surgical approach and it may be always performed in patients with isolated AS and low surgical risk, or in the case where any other associated cardiac surgery is indicated.

\section{Conclusions}

SUAVR and TAVI are both valid surgical alternatives to conventional valve replacement in patients with AS. Lower transvalvular gradients, but higher PVL, are commonly found after TAVI. Accurate preoperative screening and prosthesis selection are mandatory to properly select casespecific best treatment options, based on anatomical and surgical characteristics.

\section{Acknowledgements}

None.

\section{Footnote}

Conflicts of Interest: Dr. A D'Onofrio is a physician proctor for Edwards Lifesciences and Symetis.

\section{References}

1. Shrestha M, Fischlein T, Meuris B, et al. European multicentre experience with the sutureless Perceval valve: 
clinical and haemodynamic outcomes up to 5 years in over 700 patients. Eur J Cardiothorac Surg 2016;49:234-41.

2. Martínez-Comendador J, Castaño M, Gualis J, et al. Sutureless aortic bioprosthesis. Interact Cardiovasc Thorac Surg 2017;25:114-21.

3. D'Onofrio A, Gerosa G. Shifting a Paradigm of Cardiac Surgery: From Minimally Invasive to Micro-Invasive. J Heart Valve Dis 2015;24:528-30.

4. Leon MB, Smith CR, Mack M, et al. Transcatheter Aortic-Valve Implantation for Aortic Stenosis in Patients Who Cannot Undergo Surgery. N Engl J Med 2010;363:1597-607.

5. Adams DH, Popma JJ, Reardon MJ, et al. Transcatheter Aortic-Valve Replacement with a Self-Expanding Prosthesis. N Engl J Med 2014;370:1790-8.

6. Nishimura RA, Otto CM, Bonow RO, et al. 2017 AHA/ ACC Focused Update of the 2014 AHA/ACC Guideline for the Management of Patients With Valvular Heart Disease. J Am Coll Cardiol 2017;70:252-89.

7. Baumgartner H, Falk V, Bax JJ, et al. 2017 ESC/EACTS Guidelines for the management of valvular heart disease: The Task Force for the Management of Valvular Heart Disease of the European Society of Cardiology (ESC) and the European Association for Cardio-Thoracic Surgery (EACTS). Eur Heart J 2017. [Epub ahead of print].

8. Leon MB, Smith CR, Mack MJ, et al. Transcatheter or Surgical Aortic-Valve Replacement in Intermediate-Risk Patients. N Engl J Med 2016;374:1609-20.

9. D'Onofrio A, Salizzoni S, Rubino AS, et al. The rise of new technologies for aortic valve stenosis: A comparison of sutureless and transcatheter aortic valve implantation. J Thorac Cardiovasc Surg 2016;152:99-109.e2.

10. Muneretto C, Alfieri O, Cesana BM, et al. A comparison of conventional surgery, transcatheter aortic valve replacement, and sutureless valves in "real-world" patients with aortic stenosis and intermediate- to high-risk profile. J Thorac Cardiovasc Surg 2015;150:1570-7.

11. Miceli A, Gilmanov D, Murzi M, et al. Minimally invasive aortic valve replacement with a sutureless valve through a right anterior mini-thoracotomy versus transcatheter aortic valve implantation in high-risk patients. Eur J Cardiothorac Surg 2016;49:960-5.

12. Kamperidis V, Van Rosendael PJ, De Weger A, et al. Surgical Sutureless and Transcatheter Aortic Valves Hemodynamic Performance and Clinical Outcomes in Propensity Score-Matched High-Risk Populations With Severe Aortic Stenosis. JACC Cardiovasc Interv
2015;8:670-7.

13. Wang N, Tsai YC, Niles N, et al. Transcatheter aortic valve implantation (TAVI) versus sutureless aortic valve replacement (SUAVR) for aortic stenosis: a systematic review and meta-analysis of matched studies. J Thorac Dis 2016;8:3283-93.

14. Dionne PO, Poulin F, Bouchard D, Early Hemodynamic Results in Patients With Small Aortic Annulus After Aortic Valve Replacement. Innovations (Phila) 2017;12:254-8.

15. Belluschi I, Moriggia S, Giacomini A. et al. Can Perceval sutureless valve reduce the rate of patient-prosthesis mismatch? Eur J Cardiothorac Surg 2017;51:1093-9.

16. Theron A, Gariboldi V, Grisoli D, et al. Rapid Deployment of Aortic Bioprosthesis in Elderly Patients With Small Aortic Annulus. Ann Thorac Surg 2016;101:1434-41.

17. Theron A, Ravis E, Grisoli D, et al. Rapid-deployment aortic valve replacement for severe aortic stenosis: 1-year outcomes in 150 patients. Interact Cardiovasc Thorac Surg 2017;25:68-74.

18. Glauber M, Lio A, Miceli A. The gray zone is always more dark. J Thorac Cardiovasc Surg 2016;152:110-1.

19. Kodali SK, Williams MR, Smith CR, et al. Two-Year Outcomes after Transcatheter or Surgical Aortic-Valve Replacement. N Engl J Med 2012;366:1686-95.

20. Mack MJ, Leon MB, Smith CR, et al. 5-year outcomes of transcatheter aortic valve replacement or surgical aortic valve replacement for high surgical risk patients with aortic stenosis (PARTNER 1): a randomised controlled trial. Lancet 2015;385:2477-84.

21. Colli A, Besola L, Salizzoni S, et al. Does pre-existing aortic regurgitation protect from death in patients who develop paravalvular leak after TAVI? Int J Cardiol 2017;233:52-60.

22. Ruiz CE, Jelnin V, Kronzon I, et al. Clinical Outcomes in Patients Undergoing Percutaneous Closure of Periprosthetic Paravalvular Leaks. JACC 2011;58:2210-17.

23. Takagi H, Umemoto T; ALICE (All-Literature Investigation of Cardiovascular Evidence) Group. Prosthesis-Patient Mismatch After Transcatheter Aortic Valve Implantation. Ann Thorac Surg 2016;101:872-80.

24. Zegdi R, Bruneval P, Blanchard D, et al. Evidence of leaflet injury during percutaneous aortic valve deployment. Eur J Cardiothorac Surg 2011;40:257-9.

25. Della Barbera M, Valente M, Basso C, et al. Pericardial traumatic injury in trans-catheter aortic valve implantation. Eur J Cardiothorac Surg 2013;43:493-4.

26. van Kesteren F, Wiegerinck EMA. Autopsy after 
transcatheter aortic valve implantation. Virchows Arch 2017;470:331-9.

27. Amahzoune B, Bruneval P, Allam B, et al. Traumatic leaflet injury during the use of percutaneous valves: a comparative study of balloon- and self-expandable valved stents. Eur J Cardiothorac Surg 2013;43:488-93.

28. Foroutan F, Guyatt GH, Otto CM, et al. Structural valve deterioration after transcatheter aortic valve implantation. Heart 2017. [Epub ahead of print].

29. David TE, Feindel CM, Bos J, et al. Aortic valve replacement with Toronto SPV bioprosthesis: optimal patient survival but suboptimal valve durability. J Thorac Cardiovasc Surg 2008;135:19-24.

30. Santarpino G, Pfeiffer S, Jessl J, et al. Sutureless replacement versus transcatheter valve implantation in aortic valve stenosis: A propensity-matched analysis of 2 strategies in high-risk patients. J Thorac Cardiovasc Surg 2014;147:561-7.

31. D'Onofrio A, Rizzoli G, Messina A, et al. Conventional surgery, sutureless valves, and transapical aortic valve replacement: what is the best option for patients with aortic valve stenosis? A multicenter, propensity-matched analysis. J Thorac Cardiovasc Surg 2013;146:1065-70;

Cite this article as: D'Onofrio A, Fabozzo A, Gerosa G. Comparison of hemodynamic and clinical outcomes of transcatheter and sutureless aortic bioprostheses: how to make the right choice in intermediate risk patients. Ann Cardiothorac Surg 2017;6(5):510-515. doi: 10.21037/acs.2017.09.04 discussion 1070-1.

32. Zaman S, McCormick L, Gooley R, et al. Incidence and predictors of permanent pacemaker implantation following treatment with the repositionable LotusTM transcatheter aortic valve. Catheter Cardiovasc Interv 2017;90:147-54.

33. Nazif TM, Dizon JM, Hahn RT, et al. Predictors and clinical outcomes of permanent pacemaker implantation after transcatheter aortic valve replacement: the PARTNER (Placement of AoRtic TraNscathetER Valves) trial and registry. JACC Cardiovasc Interv 2015;8:60-9.

34. Baron SJ, Arnold SV, Wang K, et al. Health Status Benefits of Transcatheter vs Surgical Aortic Valve Replacement in Patients With Severe Aortic Stenosis at Intermediate Surgical Risk: Results From the PARTNER 2 Randomized Clinical Trial. JAMA Cardiol 2017;2:837-45.

35. Santarpino G, Pfeiffer S, Jessl J, et al. Clinical Outcome and Cost Analysis of Sutureless Versus Transcatheter Aortic Valve Implantation With Propensity Score Matching Analysis. Am J Cardiol 2015;116:1737-43.

36. Reynolds MR, Lei Y, Wang K, et al. Cost-Effectiveness of Transcatheter Aortic Valve Replacement With a SelfExpanding Prosthesis Versus Surgical Aortic Valve Replacement. J Am Coll Cardiol 2016;67:29-38. 